\title{
Electron acceleration in solar flares: observations versus numerical simulations
}

\author{
Arnold O. Benz, Paolo C. Grigis and Marco Battaglia \\ Institute of Astronomy, ETH Zurich, 8092-Zurich, Switzerland \\ email: benz@astro.phys.ethz.ch
}

\begin{abstract}
We use RHESSI hard X-ray observations to constrain acceleration of solar flare electrons, generally considered to be a primary recipient of the released energy.
\end{abstract}

Coronal X-ray sources have been previously discovered and tentatively associated with bremsstrahlung emission near the acceleration site. Now, RHESSI imaging spectroscopy (Lin et al. 2002) can temporally resolve the non-thermal spectrum of the coronal source for the first time (Battaglia \& Benz 2007). We compare the time behaviour with the predictions of stochastic acceleration, as described by transit-time damping of MHD turbulence excited by reconnection. The results in five limb events indicate Soft-HardSoft (SHS) behaviour of the coronal source emission in the course of an X-ray peak (the more flux, the harder the spectrum, Grigis \& Benz 2004; Battaglia et al. 2005). The SHS behaviour thus constitutes a conspicuous property of the acceleration process. The temporal behaviour of the spectrum can be quantitatively described by a pivot point at a photon energy of about $20 \mathrm{keV}$ at which the flux remains constant in time.

We solve a diffusion equation for the interaction of waves and particles including trapping, escape and particle replenishment (Grigis \& Benz 2006). The solution yields a spectrum that is approximately a power-law in the observed range of energies. However, the theoretically derived pivot point is generally at energies lower than observed. For this reason we include transport effects, such as produced by an electric potential, or scattering in the coronal source (collisional trapping) to bring the pivot energy up to the observed value. Escaping particles propagate to the base of the loop in the dense chromosphere. These precipitating particles are identified as origin of the observed hard X-ray footpoints.

Observations and simulations show that solar flare electron acceleration cannot be modeled without transport effects. The observations are consistent with stochastic acceleration in a relatively dense medium (up to $10^{11} \mathrm{~cm}^{-3}$ ) high wave energy densities (up to 0.001 magnetic) and a return current. The observations also constrains the global flare geometry, requiring coupling between the coronal source and the chromospheric footpoints.

Acknowledgments. The analysis of RHESSI data at ETH Zurich is partially supported by the Swiss National Science Foundation (grant nr. 20-67995.02).

\section{References}

Battaglia, M., \& Benz, A.O. 2007, A\& A, 466, 713

Battaglia, M., Grigis, P.C., \& Benz, A.O. 2005, A\&A 439, 737

Grigis, P.C., \& Benz, A.O. 2004, A $\& A 426,1093$

Grigis, P.C., \& Benz, A.O. 2006, A\& $A, 458,641$

Lin, R.P., Dennis, B.R., Hurford, G.J., et al. 2002, Solar Phys., 210, 3 